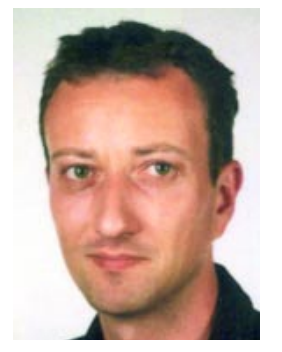

Christoph Dehnicke

\title{
Epilepsiechirurgie
}

\section{Was passiert Wann? Wie? Wo? Warum? Und mit welchen Ergebnissen?}

Christoph Dehnicke, Heinz-Joachim Meencke Epilepsie-Zentrum Berlin-Brandenburg im Verbund der v. Bodelschwinghschen Anstalten Bethel, Evangelisches Krankenhaus Königin Elisabeth Herzberge, Akademisches Lehrkrankenhaus der Charité Berlin

psychoneuro 2004; 30 (2): 83-86

$\mathrm{E}$ pilepsien haben mit ca. 0,5-1\% die höchste Prävalenz unter den chronischen neurologischen Erkrankungen. Bei der genauen Zuordnung zu einem Epilepsiesyndrom wird unter anderem zwischen fokalen und generalisierten Epilepsien unterschieden. Der Anteil von Menschen mit fokalen Epilepsien ist mit 60-70\% etwas höher als der mit generalisierten Epilepsiesyndromen.

Für die Behandlung kommen zunächst für alle Epilepsiesyndrome dieselben therapeutischen Möglichkeiten in Frage. Hierzu gehören medizinische, psychologische, soziale und berufliche Ansatzpunkte. Ziel der Epilepsiebehandlung ist die Anfallsfreiheit, ohne therapiebedingte körperliche und geistige Beeinträchtigung, mit individuell akzeptierter Lebensqualität. Die Grundlage jeder medizinischen Epilepsiebehandlung ist die medikamentöse Therapie, für die eine Reihe von Wirkstoffen zur Verfügung steht. Nach konsequent durchgeführter medikamentöser Behandlung kann bei bis zu 90\% der idiopathisch generalisierten Epilepsien Anfallsfreiheit erzielt werden. Demgegenüber kann bei Menschen mit fokalen Epilepsien, je nach Syndrom, nur in

Haben bei einer fokalen Epilepsie zwei Standard-Antiepileptika nicht zur Anfallsfreiheit geführt, muss die Möglichkeit einer operativen Therapie überprüft werden. Die präoperative Diagnostik umfasst die Ergebnisse der Untersuchungen eines multiprofessionellen Teams bestehend aus Epileptologen, Neuropsychologen, Psychiatern und Sozialarbeitern. Ergebnis der nichtinvasiven Untersuchungsverfahren sind vier fokale Epilepsiesyndrome mit unterschiedlicher Prognose bezüglich der zu erwartenden Anfallsfreiheit. In bestimmten Fällen, die sehr individuell entschieden werden müssen, ist eine invasive Diagnostik erforderlich. Im Vergleich zu den Chancen bei alleiniger Weiterführung der medikamentösen Therapie mit weniger als 20\% liegt die Chance auf Anfallsfreiheit durch eine operative Therapie in Abhängigkeit von den Syndromen zwischen 50 und 85\%.

60-70\% der Fälle eine Anfallsfreiheit mit Hilfe der medikamentösen Therapie und den oben genannten flankierenden therapeutischen Maßnahmen erreicht werden (9).

\section{Für wen kommt eine epilepsiechirurgische Therapie in Frage?}

Um über einen epilepsiechirurgischen Eingriff Anfallsfreiheit erzielen zu können, ist es notwendig, die so genannte „epileptogene Zone“ zu ermitteln und zu entfernen. Definitionsgemäß handelt es sich hierbei um „das Kortexareal, welches nötig und in der Lage ist, epileptische Anfälle auszulösen und dessen Entfernung notwendig ist, um Anfallsfreiheit zu erzielen“. Zur Ein- grenzung dieser Zone werden mit Hilfe verschiedener Untersuchungsverfahren strukturelle und funktionelle Läsionen lokalisiert. Bei generalisierten Epilepsien lassen sich grundsätzlich keine eng umschriebenen strukturellen und funktionellen Läsionen ermitteln, so dass für einen epilepsiechirurgischen Eingriff nur Patienten mit einem fokalen Epilepsiesyndrom in Frage kommen. Eine weitere Bedingung, die erfüllt sein muss, ist die „Pharmakoresistenz“. Nach einer Definition der Arbeitsgemeinschaft Epilepsiechirurgie liegt Pharmakoresistenz vor, wenn: „zwei Medikamente der ersten Wahl in Mono- oder Kombinationstherapie nicht zur Anfallsfreiheit führen“. 


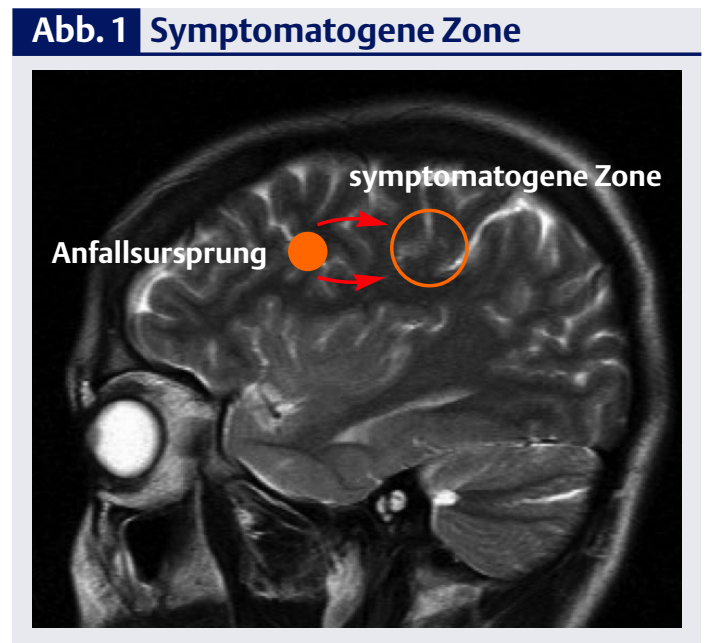

Rote Pfeile: Ausbreitung der Anfallsaktivität mit zeitlicher Latenz

\section{Was ist erforderlich?}

Welche diagnostischen Maßnahmen sind vor einem epilepsiechirurgischen Eingriff erforderlich und welche Informationen liefern uns die einzelnen Verfahren? Vor jedem epilepsiechirurgischen Eingriff muss eine umfangreiche präoperative Diagnostik durchgeführt werden. Insgesamt dient sie der Auffindung und Lokalisation von strukturellen und funktionellen Störungen. Über die Zusammenschau der Ergebnisse voneinander unabhängiger Untersuchungsverfahren wird die „epileptogene Region“ ermittelt. Im Einzelnen

\section{Abb. 2 Epilepsiesyndrome}

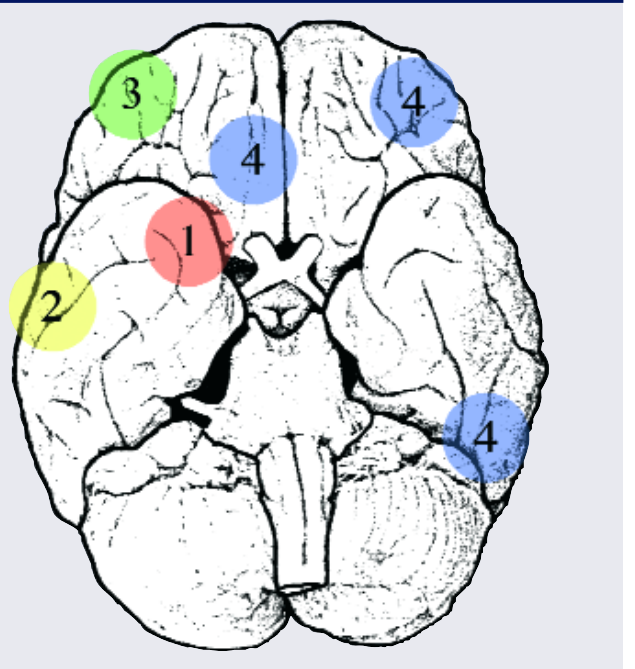

Die vier Epilepsiesyndrome nach Abschluss der präoperativen Diagnostik: 1. mesio-temporal; 2. temporo-lateral; 3.extratemporal; 4. bilateral/multifokal werden folgende Untersuchungsverfahren angewendet:

\section{Klinische Untersuchungen}

Allgemeine und spezielle neurologische Anamneseerhebung und klinische neurologische Untersuchung, neuropsychologische Untersuchung, psychiatrische Evaluation und Sozialanamnese. Die Datenerhebung wird von Personen der einzelnen Fachbereiche mit Zusatzqualifikationen auf dem Gebiet der präoperativen Diagnostik durchgeführt.

\section{Apparative Diagnostik}

Strukturelle Bildgebung: Die MRT (Magnet-Resonanz Tomographie) gehört mittlerweile zum allgemeinen Standard der Diagnostik bei Epilepsien. Im Rahmen der präoperativen Diagnostik werden jedoch besondere Anforderungen an das Bild gestellt. Die Aufnahmen erfolgen in verschiedenen Sequenzen, Gewichtungen (T1, T2, IR, FLAIR) und Messverfahren (Volumetrie, Relaxometrie, Voxel-Based-Morphometry). Die Frage, ob die mit Hilfe des MRTs im Einzelfall gefundenen strukturellen Auffälligkeiten auch in einem ursächlichen Zusammenhang mit der Epilepsie stehen, muss durch ein Video-EEG-Intensivmonitoring geklärt werden.

Funktionelle Bildgebung: Hierzu gehören PET (Positronen Emissions Tomography), iktales SPECT (Single Photon Emission Computed Tomography) und fMRT (funktionelles MRT). PET und SPECT dienen der Auffindung von regionalen funktionellen Störungen, wobei verschiedene Substrate des neuronalen Stoffwechsels und Transmittersubstanzen eingesetzt werden können. Mit Hilfe des fMRTs hingegen werden Regionen kenntlich gemacht, in denen sich Sprach-, Gedächtnisoder motorische Funktionen befinden.

Video-EEG-Intensivmonitoring: Wichtigste Untersuchung im Rahmen der präoperativen Diagnostik ist das Video-EEG-Intensivmonitoring mit dichtgesetzten Oberflächen- und Sphenoidalelektroden, da das EEG die einzige Untersuchungsmethode ist, die in der Lage ist, die elektrophysiologischen Vorgänge während eines Anfalls darzustellen. Ziel ist es, die habituellen Anfälle des Patienten aufzuzeichnen. Zur „Anfallsprovokation" können im Vorfeld die Antikonvulsiva reduziert werden. Über die Einzelanalyse der Video- und EEG-Daten können Informationen über die Lokalisation des Anfallsursprungs gewonnen werden. Der klinisch sichtbare Verlauf des Anfalls (Anfallssemiologie) oder das vom Patienten selbst bemerkte Anfallsgeschehen (Auren) geben uns Auskunft über die Lokalisation der „symptomatogenen Zone“. Dies ist das Kortexareal, in dem sich die Anfallsaktivität zum Zeitpunkt des Auftretens der Anfallssymptomatik befindet. Es bedeutet jedoch nicht, dass der Anfall auch dort seinen Ursprung hat, da der Anfall auch in einem „klinisch stummen“ Kortexareal entstanden sein kann. In diesem Fall läuft die Anfallsaktivität schon eine Weile ab und es kommt erst zu klinischen Auffälligkeiten, wenn sie sich in eloquente Kortexareale ausgebreitet hat (Abb. 1). Der zeitliche Zusammenhang zwischen Beginn des EEG-Anfallsmusters und Beginn des klinischen Anfallsgeschehens (EEG-Klinik-Korrelation) gibt einen Hinweis auf die Verwertbarkeit der Lokalisation des Initialen EEG-Anfallsmusters (3).

Beginnt das EEG-Anfallsmuster vor dem klinischen Anfallsbeginn, kann ein wesentlich engerer räumlicher Zusammenhang zwischen der Region des EEG-AM und anfallsauslösender Region angenommn werden, als wenn das EEG-AM erst sichtbar wird, wenn der Anfall klinisch schon abläuft, bevor ein Anfallsmuster im EEG sichtbar wird. Bei guter EEG-Klinik-Korrelation gibt die Lokalisation des Beginns der EEG-AM von allen nichtinvasiven Untersuchungsmethoden den sichersten Hinweis auf die Lokalisation der anfallsauslösenden Region.

\section{Wie und Wann wird über eine Operation entschieden? Nach Abschluss des Video-EEG- Intensivmonitorings wird in der Re- gel im Rahmen einer interdiszi- plinären Fallkonferenz eine Entschei- dung über das weitere Vorgehen ge-}


troffen, insbesondere eine RisikoNutzen-Abwägung vorgenommen.

Das nichtinvasive Video-EEGIntensivmonotoring führt zur Abgrenzung von vier klinisch-elektroenzephalographischen Syndromen: die mesiotemporalen, die temporolateralen, die extratemporalen und bilateral/multifokalen Syndrome (Abb. 2).

Diese vier Syndrome erfordern unterschiedliche Vorgehensweisen. Die mesiotemporalen Epilepsien, bei denen in der Bildgebung zwischen 60 und $70 \%$ Hinweise auf Hippokampussklerosen nachweisbar sind, sind das häufigste chirurgisch behandelte Syndrom, das keine invasive Abklärung erfordert. Zur Risiko-NutzenAbwägung muss insbesondere der Neuropsychologe etwas zum Risiko postoperativer Gedächtnis- und Sprachdefizite aussagen (siehe auch Beitrag von Hättig in diesem Heft).

Neben den neuropsychologischen Befunden gehen Sozialdaten und Daten der psychiatrischen Untersuchung in die Einschätzung der Risikoprognose ein. Psychiatrische Probleme sind bei Patienten mit pharmakoresistenter Epilepsie nicht selten. Häufig werden postoperativ depressive Verstimmungen beobachtet. Über das Risiko sollte der Patient im Vorfeld aufgeklärt werden. Diese depressiven Störungen sind gut behandelbar und in der Regel spätestens ein Jahr nach der Operation abgeklungen. Postoperativ neu auftretende Psychosen sind extrem selten. Allerdings stellen präoperative psychiatrische Auffälligkeiten einen Risikofaktor dar.

An Hand der Sozialdaten sollte möglichst schon präoperativ ein Rehabilitationsplan erstellt werden, dessen Umsetzung dann in der sich anschließenden Anschluss-Heilbehandlung in einem auf Epilepsie spezialisierten Rehazentrum eingeleitet werden kann. Die weitere Realisierung muss dann während der regelmäßigen Nachbetreuung durch den Sozialdienst des Epilepsiezentrums erfolgen $(2,7)$.

\section{Welche invasiven EEG- Methoden gibt es und wann werden sie angewendet?} Foramen ovale und peg-Elektroden: Durch die verbesserte bild-

\section{Abb. 3 Röntgenbilder des Schädels mit implantierten Plattenelektroden}

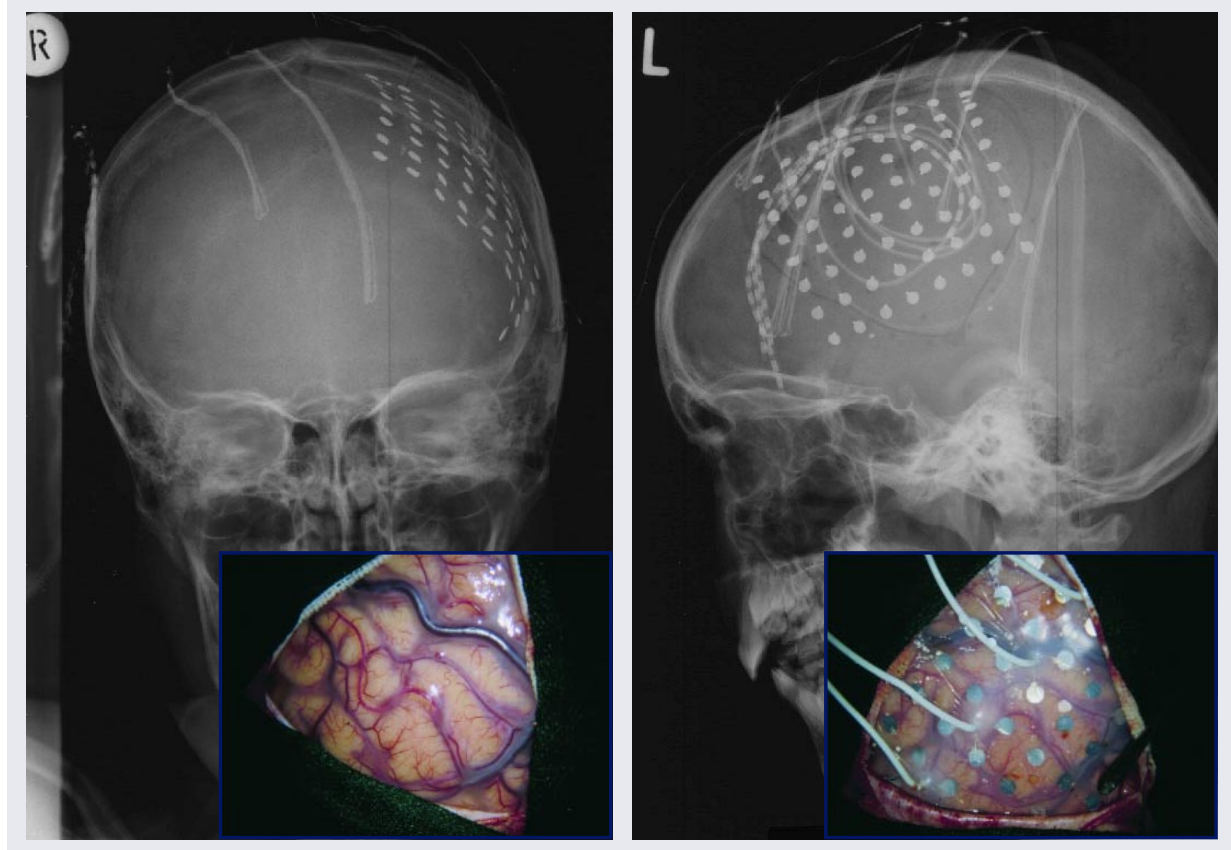

Unten: Operationssitus links ohne, rechts mit Plattenelektroden

gebende Diagnostik hat die Notwendigkeit, invasive Untersuchungsmethoden einzusetzen, deutlich abgenommen. Bei unklarer Lateralisation des Anfallsursprungs (nicht lateralisierte Anfallsmuster im Oberflächen-EEG oder diskrepante Befunde zwischen den verschiedenen Untersuchungsverfahren; EEG, MRT, PET) nach dem Monitoring mit Oberflächenelektroden, der Notwendigkeit der weiteren Eingrenzung des Anfallsursprungs (Ausmaß der Resektion) oder sicheren Unterscheidung von mesio-temporalen und temporo-lateralem Anfallsursprung (unterschiedliches neurochirurgisches Vorgehen) kommen foramen ovale und peg-Elektroden zum Einsatz.

Subdurale Plattenelektroden: Diese Elektroden werden verwendet, wenn eine weitere Eingrenzung der epileptogenen Region und Abgrenzung zum eloquenten Kortex erforderlich ist. Dies ist insbesondere bei einer epileptogenen Region temporolateral auf der sprachdominanten Seite notwendig. Die Einlage der Elektroden erfolgt subdural über eine Trepanation (Abb. 3).

Nach der Implantation erfolgt neben dem Video-EEG-Monitoring zur Aufzeichnung von Anfällen eine kortikale Stimulationsuntersuchung zur Bestimmung funktionell relevanter Areale. Es erfolgt eine exakte „Kartierung“ von epileptogenen Regionen und funktionell relevanten Kortexarealen nach der dann die Resektionsgrenzen bestimmt werden können (Abb. 4).

Tiefenelektroden: Stereotaktisch gesetzte Tiefenelektroden werden nur noch sehr selten bei besonderen Konstellationen bitemporaler Epilepsien eingesetzt (sind da aber häufig durch foramen ovale- und peg-Elektroden ersetzbar) oder wenn Ausbreitungsphänomene im Makronetzwerk der Epilepsie untersucht werden müssen.

\section{Aspekte des prä- und post- operativen Verlaufs}

Die präoperative Epilepsiediagnostik und operative Epilepsietherapie gehört in die Hände eines erfahrenen Teams. Die Risiko-NutzenAbwägung muss immer für den Einzelfall individuell erfolgen. Ein Problem weltweit ist, dass Patienten mit durchschnittlich 15 Jahren Krankheitsverlauf zu spät zur Abklärung einer chirurgischen Behandlungsmöglichkeit in ein Epilepsiezentrum überwiesen werden. Es sollte innerhalb von zwei Jahren 


\section{Abb. 4 Ergebnisse einer Untersuchung mit subduralen Plattenelektroden}
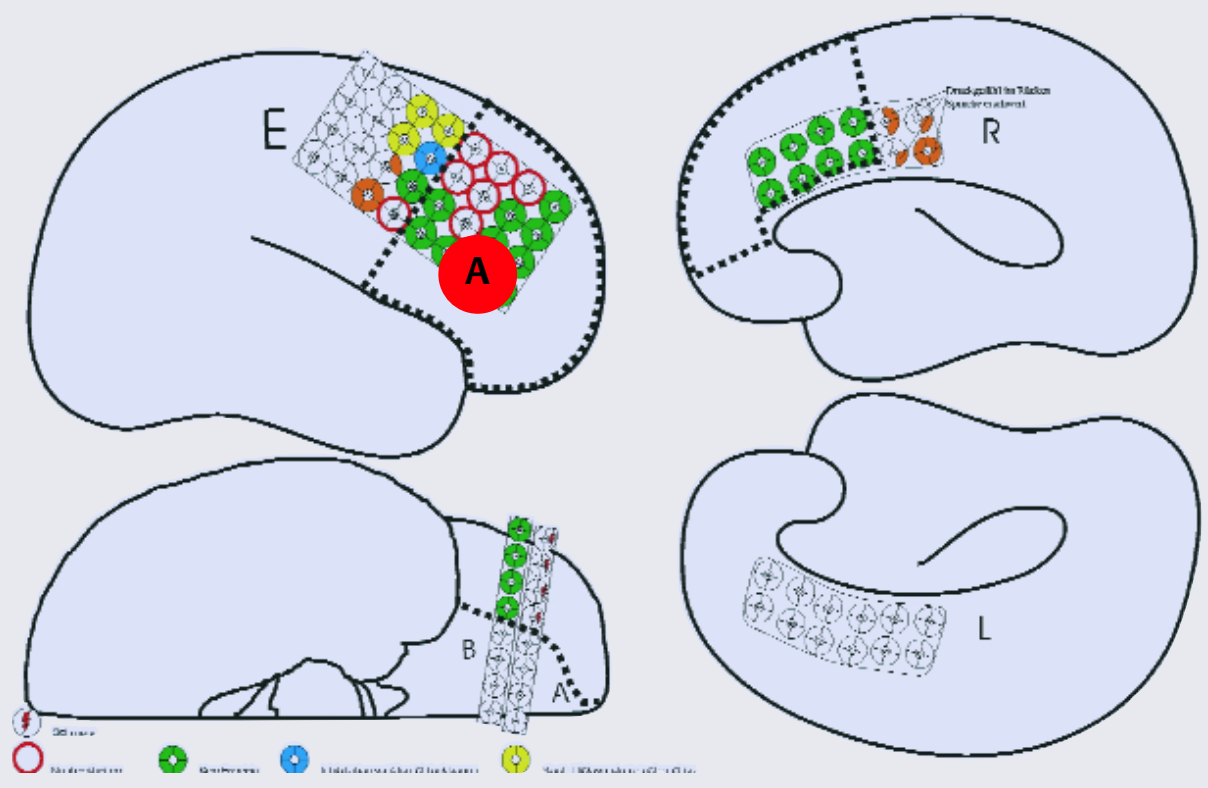

Roter Kreis mit A: Lokalisation des initialen EEG-Anfallsmusters; orange, blau, gelbe Elektroden: motorische Funktionen; grüne Elektroden: keine Ereignisse; rot umrandet: Nachentladungen; gestrichelte Linie: Resektionsgrenzen

nach Erkrankungsbeginn die Wirksamkeit von zwei Standard-Antiepileptika überprüft werden. Bei Versagen sollte schon zu diesem Zeitpunkt geklärt werden, ob eine epilepsiechirurgische Option besteht, da die Chance durch ein weiteres Medikament anfallsfrei zu werden unter 20\% liegt. Demgegenüber können Patienten mit einer mesiotemporalen Epilepsie bei Hippokampussklerose mit 70-85\% Chance auf Anfallsfreiheit rechnen. Bei neokortikalen und extratemporalen Epilepsien liegt die Chance nur zwischen 50 und $60 \%$, weil häufig wegen der Überschneidung von epileptogener Region und eloquentem Kortex die epileptogene Region nicht komplett reseziert werden kann. Die Ergebnisse dieser Gruppe von Epilepsien ist aber um so besser, je klarer ein morphologischer Befund nachweisbar ist.

Über diese Chancen muss ein Patient frühzeitig bei der Therapieplanung aufgeklärt werden. In unserem Zentrum haben sich in Zusammenarbeit mit der Selbsthilfe Diskussionsgruppen bewährt, die operierte Patienten und Patienten, die vor der Entscheidung zu einer Operation stehen, zusammenbringt und einen Erfahrungsaustausch möglich machen $(1,2,5,6,8,10)$.

Seizure freedom is the aim of all treatment in epilepsy patients. The options of a surgical treatment in patients with a focal epilepsy should always be evaluated if two standard antiepileptic drugs have failed in getting the patient seizure free. The presurgical work-up takes into regard the results of the diagnostic evaluation by a multi-professional team which comprises of epileptologists, neuropsychologists, psychiatrists and social workers. Basically the result of the non-invasive electro-clinical work-up leads us to one of four major categories of epileptic syndroms which differ regarding their prognosis/ postoperative outcome. Some cases need to be evaluated further, using invasive electrodes. Compared to the prospects getting a patient seizure free with ongoing anti-epileptic drug treatment alone (cumulative chances being below $20 \%$ ), the chances to gain seizure freedom by means of epilepsy surgery vary between $50 \%$ and $85 \%$ dependent on the underlying syndrome.

\section{KeyWords:}

Seizure freedom - epilepsy - epilepsy

surgery - prognosis

\section{Literatur}

1. Berkovic SF, Mclntosh AM, Kalnins RM et al. Preoperative MRI predicts outcome of temporal lobectomy: an actuarial analysis. Neurology 1995; 45: 1358-1363

2. Blumer D, Wkjlu S, Davies K, Hermann B. Psychiatric outcome of temporal lobectomy: incidence and treatment of complications. Epilepsia 1998; 39: 478-486

3. Engel J Jr. Update on the surgical treatment of the epilepsies. Neurology 1993; 43 : 1612-1617

4. Engel J. Outcome with respect to epileptic seizures. In: Engel J Jr ed. Surgical treatment of the epilepsies. New York: Raven, 1993; 609-621

5. Gilliam F, Kuzniecky R, Meador $\mathrm{K}$ et al. Patient-oriented outcome assessment after temporal lobectomy for refractory epilepsy. Neurology 1999; 53: 687-694

6. Kuzniecky R, Burgard $S$ et al. Predictive value of magnetic resonance imaging in temporal lobe epilepsy surgery. Arch Neurol 1993; 50: 65-69

7. Moran PW, Lambert MJ. A review of current assessment tools for monitoring changes in depression. In: Lambert MJ, Christensen ER, DeJulio SS (eds.). The measurement of psychotherapy outcome in research and evaluation. New York: Wiley, 1983; 263-303

8. Reeves AL, So EL, Evans R. Factors associated with work outcome after anterior temporal lobectomy for intractable epilepsy. Epilepsia 1997; 38: 689-695

9. Theodore WH. What is uncontrolled epilepsy and should be referred for epilepsy surgery? In: Spencer SS, Spencer DD (eds.). Surgery for epilepsy. Cambridge: Blackwell, 1991;3-17

10. Wiebe S, Blume W, Girvin J et al. A randomized, controlled trial of surgery for temporal lobe epilepsy. N Engl J Med 2001; 345: 311-366

\section{Korrespondenzadresse}

Dr. med. Christoph Dehnicke Oberarzt

Prof. Dr. Heinz-Joachim Meencke Medizinischer Direktor

Epilepsie-Zentrum Berlin-Brandenburg im Verbund der v. Bodelschwinghschen Anstalten Bethel

Evangelisches Krankenhaus

Königin Elisabeth Herzberge

Akademisches Lehrkrankenhaus der Charité Herzbergstr. 79

10365 Berlin

cdehnicke@izzb.de 